\title{
Caracterização termogravimétrica e espectroscópica das propriedades ácidas da zeólita ZSM-22
}

\section{(Thermogravimetric and spectroscopic characterization of acidic properties of ZSM-22 zeolite)}

\author{
A. R. Loiola ${ }^{1}$, J. C. R. de A. Andrade ${ }^{1}$, J. M. Sasaki ${ }^{1}$ L. R. D. da Silva ${ }^{1}$, E. J. Nassar ${ }^{2}$ \\ ${ }^{1}$ Universidade Federal do Ceará, Av. Prof. Aurélio Câmara s/n, C.P. 6002, Campus do Pici \\ Fortaleza, CE 60451-970 \\ ${ }^{2}$ Universidade de Franca, Av. Dr. Armando Salles Oliveira 201, C.P. 82, Franca, SP 14404-600 \\ pillaredclay@yahoo.com.br,adonayrloiola@yahoo.com \\ jacarolim@yahoo.com.br,sasaki@fisica.ufc.br,ejnassar@unifran.br
}

\begin{abstract}
Resumo
A acidez da estrutura da zeólita ZSM-22 foi determinada por análise térmica. A energia de ativação, calculada pelo método de Ozawa, envolveu uma energia de termodecomposição de $18,44 \mathrm{~kJ} \cdot \mathrm{mol}^{-1}$. O valor do fator pré-exponencial A foi de 2,2746. Suas propriedades texturais foram determinadas por análise de dessorção/adsorção de nitrogênio, cujos resultados indicaram a existência de microporosidade, com área de superfície de $257 \mathrm{~m}^{2} \cdot \mathrm{g}^{-1}$, volume de microporos de $0,041 \mathrm{~cm}^{3} \cdot \mathrm{g}^{-1}$ e diâmetro de poros de $32,6 \AA$. Análises de luminescência, utilizando európio como sonda, sugerem que a acidez é distribuída uniformemente dentro da estrutura da zeólita.
\end{abstract}

Palavras-chave: zeólita ZSM-22, acidez, método de Ozawa.

Abstract

The acidity and stability of ZSM-22 zeolite structure have been studied by thermal analysis. The activation energy calculated by Ozawa method to the events related to thermodecomposition was $18.44 \mathrm{~kJ} \mathrm{~mol}^{-1}$. The value of the pre-exponential factor $\mathrm{A}$ was 2.2746. Its textural properties have been determined by nitrogen adsorption desorption analysis, whose results indicated microporous properties, with surface area $257 \mathrm{~m}^{2} \cdot \mathrm{g}^{-1}$, micropore volume $0.041 \mathrm{~cm}^{3} \cdot \mathrm{g}^{-1}$ and pore diameter 32.6 A. Luminescence analyses, using europium as probe, suggest that the acidity is distributed uniformly within the zeolite structure.

Keywords: ZSM-22 zeolite, acidit, Ozawa method.

\section{INTRODUÇÃO}

Zeólitas são aluminossilicatos microporosos com estrutura aberta, constituídas por unidades tetraédricas que originam um sistema de poros e cavidades com dimensões moleculares [1]. Tetraedros formados por $\mathrm{SiO}_{4}$ e $\mathrm{AlO}_{4}$, ligados entre si pelos átomos de oxigênio, constituem os principais componentes da maioria das zeólitas, além de outros tetraedros como os de $\mathrm{PO}_{4} \mathrm{e}^{\mathrm{GaO}}$ também representarem uma fração importante de muitas zeólitas [2]. Sua composição química pode ser convenientemente representada por $\mathrm{A}_{\mathrm{y} / \mathrm{m}}{ }^{\mathrm{m}+}\left[\left(\mathrm{SiO}_{2}\right)_{\mathrm{x}} \cdot\left(\mathrm{AlO}_{2}\right)_{\mathrm{y}}^{-}\right] \cdot \mathrm{zH}_{2} \mathrm{O}$, na qual A é o cátion com carga $m,(\mathrm{x}+\mathrm{y})$ é o número de tetraedros por unidades de célula e x:y é a razão silício:aluminio. De acordo com a bem estabelecida regra de Löwenstein, ligações Al-O-Al não são permitidas e $\mathrm{y} / \mathrm{x} \geq 1$. Muitas zeólitas ocorrem como minerais naturais, mas são suas variedades sintéticas que estão entre os materiais mais usados como adsorventes, catalisadores e trocadores iónicos [3, 4]. As zeólitas são materiais tecnologicamente importantes e que encontram aplicações em uma série de processos químicos, sendo que uma de suas propriedades mais marcantes, da qual deriva grande parte de sua funcionalidade, é sua acidez [5]. Deste modo, a natureza dos sítios ácidos (Brönsted ou Lewis) assim como sua distribuição e concentração na estrutura zeolítica são parâmetros de grande importância na caracterização de uma zeólita. A zeólita ZSM-22 é caracterizada por um sistema de canais unidimensionais. Apesar de sua estrutura apresentar anéis de 5, 6 e 10 membros, apenas os de 10 membros dão origem aos sistemas de poros já que canais pequenos não permitem o acesso e a difusão de moléculas, não sendo considerados portanto como poros [6]. Os anéis de 10 membros da zeólita ZSM-22 apresentam diâmetro livre de 0,55 x $0,44 \mathrm{~nm}[7,8]$.

\section{MATERIAIS E MÉTODOS}

As medidas de difração de raios $\mathrm{X}$ foram realizadas em um difratômetro de pó Philips. Foi usada radiação de cobre $(\mathrm{Cu}-\mathrm{k} \alpha, \lambda=1,54056 \AA)$ com tubo operando em $40 \mathrm{kV}$ 
e $40 \mathrm{~mA}$. Análises de microscopia eletrônica de varredura foram conduzidas em equipamento Philips série LX-30. Para a quantificação dos sítios ácidos, partiu-se de uma pastilha pura da zeólita ZSM-22, previamente calcinada em mufla com taxa de aquecimento $15^{\circ} \mathrm{C} \cdot \mathrm{min}^{-1}$ até $400^{\circ} \mathrm{C}$, permanecendo nesta temperatura por $2 \mathrm{~h}$. A pastilha da zeólita, após ter seu espectro obtido na região do infravermelho, foi colocada em um balão sob vácuo de aproximadamente $10^{-3} \mathrm{~atm}$ e aquecido a $250^{\circ} \mathrm{C}$ por $2 \mathrm{~h}$, objetivando a eliminação de umidade retida na sua estrutura. Após um curto intervalo de tempo, onde se verificou a estabilização da temperatura, fechou-se o balão e, ficando evacuado, conectou-se este a um recipiente dentro do qual se encontrava $1 \mathrm{~mL}$ de piridina. A diferença de pressão entre os dois recipientes proporcionou a passagem de vapor de piridina através da pastilha de zeólita. A pastilha foi mantida sob vácuo por $30 \mathrm{~min}$ para eliminar o excesso de piridina. Em seguida, obteve-se o seu espectro na região do infravermelho utilizando espectrômetro Shimadzu IR Prestige 21. A pastilha foi então calcinada sucessivamente a $100,200,300$ e $400{ }^{\circ} \mathrm{C}$ durante $1 \mathrm{~h}$. Cada aquecimento foi seguido por resfriamento em dessecador e análise espectral na região do infravermelho. As análises espectroscópicas da zeólita com piridina foram usadas para investigar a acidez. A área das bandas características de interação entre a piridina e a zeólita foram usadas para calcular a acidez de cada sítio, Brönsted e Lewis, por meio da equação [9]:

$$
\mathrm{q}_{\mathrm{B}, \mathrm{L}}=\left(\mathrm{A}_{\mathrm{B}, \mathrm{L}} \pi \mathrm{D}^{2}\right)\left(4 \mathrm{wE}_{\mathrm{B}, \mathrm{L}}\right)^{-1}
$$

na qual $\mathrm{D}=$ diâmetro da pastilha $(\mathrm{cm}) ; \mathrm{w}=$ massa da amostra $(\mathrm{g}) ; \mathrm{A}_{\mathrm{B}, \mathrm{L}}=$ integração das áreas das bandas características (absorbância), obtido com auxílio de software adequado após otimização da linha de base; $\mathrm{E}_{\mathrm{B}, \mathrm{L}}=$ coeficiente de extinção da interação de piridina com os sítios ácido de Brönsted $=1,67$ $\pm 0,12 \mathrm{~cm} \cdot \mu \mathrm{mol}^{-1}$ e Lewis $=2,22 \pm 0,21 \mathrm{~cm} \cdot \mu \mathrm{mol}^{-1}$.

As análises termogravimétricas foram realizadas em equipamento Shimadzu TGA-50H, usando quatro amostras de massa $\sim 7,6 \mathrm{mg}$, aquecidas a taxas de $2,5,7$ e $10^{\circ} \mathrm{C} \cdot \mathrm{min}^{-1}$, em recipiente de platina, em atmosfera de ar com fluxo de $50 \mathrm{~mL} \cdot \mathrm{min}^{-1}$. A energia de ativação para as perdas de massas na termogravimetria foi calculada pelo método de Ozawa [10], que estabelece que a massa fracionária (W) de um material reacional é expressa como função quantitativa de sua estrutura como um grupo, um constituinte, uma quebra de ligação etc., a qual é representada por: $\mathrm{W}=\mathrm{f}(\mathrm{x})$, onde $\mathrm{x}$ varia de acordo com a equação cinética ordinária:

$$
-\int_{\mathrm{x}_{0}}^{\mathrm{x}} \frac{\mathrm{dx}}{\mathrm{dt}}=\mathrm{A} \int_{\mathrm{t}_{0}}^{\mathrm{t}} \mathrm{e}^{\mathrm{Ea} / \mathrm{RT}} \mathrm{g}(\mathrm{x})
$$

A excitação e os espectros de emissão foram obtidos com um espectrofluorímetro Spex Fluorolog II, na temperatura ambiente $\left(25^{\circ} \mathrm{C}\right)$ e na temperatura do nitrogênio líquido $\left(-196{ }^{\circ} \mathrm{C}\right)$, respectivamente. As amostras foram colocadas em um tubo capilar de quartzo (diâmetro interno 1,0 mm). A emissão foi coletada em $90^{\circ} \mathrm{em}$ relação ao feixe de excitação. As fendas foram colocados em 2,0 e $0,5 \mathrm{~mm}$ para excitação e emissão, respectivamente, dando uma banda de resolução
7 e 0,9 nm. Filtros Oriel 59816 (excitação) e Corning 97612 (emissão) foram utilizados.

Para determinação das propriedades texturais utilizou-se sistema de adsorção/dessorção de nitrogênio. Uma alíquota de $0,1940 \mathrm{~g}$ da zeólita calcinada e padronizada em peneira de 200 mesh foi introduzida ao sistema de adsorção/dessorção de $\mathrm{N}_{2}$ por $1 \mathrm{~h}$, com temperatura de dessorção de $110{ }^{\circ} \mathrm{C}$. Foram utilizados os métodos de multipontos BET e da curva de Langmuir.

\section{RESULTADOS E DISCUSSÃO}

Os resultados obtidos a partir do refinamento usando o método de Rietveld no padrão de difração da zeólita ZSM-22 são mostrados na Fig. 1. Estes dados indicam que o material pertence ao grupo espacial $\mathrm{Cmcm}$, e apresenta simetria ortorrômbica. Os parâmetros de célula são: $a=$ $13,818 \AA \pm 0,001, \mathrm{~b}=17,377 \AA \pm 0,003 \mathrm{e} \mathrm{c}=5,0296 \AA$ $\pm 0,005$. As intensidades dos picos de difração observados nos padrões são um pouco diferentes dos observados no difratograma calculado. Entretanto, observa-se que a posição dos picos condiz com a simetria ortorrômbica. Os cristais zeolíticos apresentaram tamanhos, calculados a partir da equação de Scherrer, de 31, 33 e $32 \mathrm{~nm}$. Estes valores foram determinados levando-se em consideração três diferentes planos cristalográficos: (100), (400) e (111), respectivamente, do padrão de difração da zeólita. Estes resultados são corroborados pelas informações oriundas das análises de microscopia eletrônica, Fig. 2. A morfologia dos cristais da zeólita ZSM-22 indica formas e tamanhos regulares.

A composição química da zeólita ZSM-22 pode ser representada pela fórmula $\left(\mathrm{NH}_{4}^{+}\right)\left[\left(\mathrm{SiO}_{2}\right)_{46}\left(\mathrm{AlO}_{2}^{-}\right)\right] \cdot \mathrm{zH}_{2} \mathrm{O}$. Dentre os parâmetros essenciais está a acidez, uma vez que importantes propriedades derivam dos tipos de sítios ácidos, assim como de suas concentrações e distribuições.

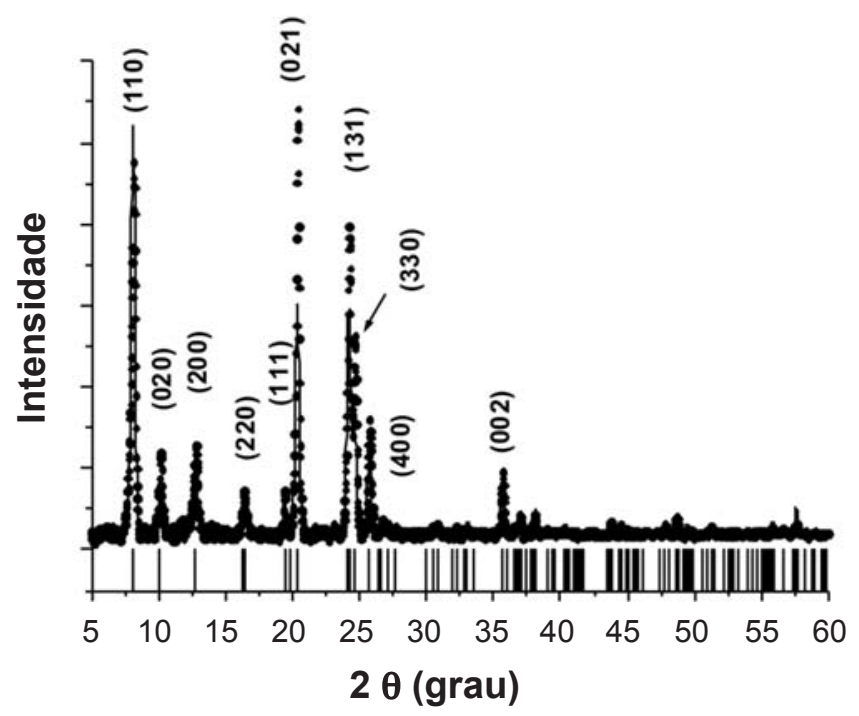

Figura 1: Difratograma de raios X experimental (ponto) e calculado (linha) da Zeólita ZSM-22.

[Figure 1: Experimental (point) and calculated (line) XRD patterns of the ZSM-22 zeolite.] 


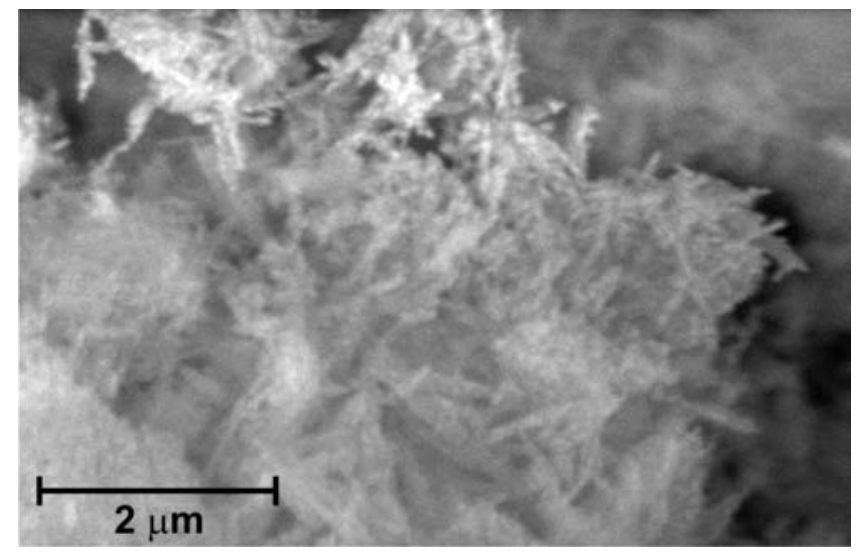

Figura 2: Micrografia obtida em microscópio eletrônico de varredura da zeólita, mostrando os cristalitos.

[Figure 2: Scanning electron microscopy micrograph of the obtained ZSM-22 zeolite.]

A acidez de Brönsted deve decorrer prioritariamente dos sítios de coordenação do íon amônio. Estudos sugerem que esta acidez se manifesta nas unidades pentasil da zeólita, indicando assim a localização preferencial dos contra-íons [11]. Um efeito provocado pela ativação térmica confere a esta zeólita uma característica adicional. Entre 300 e $400{ }^{\circ} \mathrm{C}$ deve ocorrer a liberação de espécies $\mathrm{H}^{+}$em decorrência da termodegradação dos íons amônio e conseqüente liberação de amônia $\left(\mathrm{NH}_{4}^{+}{ }^{\circledR} \mathrm{NH}_{3(\mathrm{~g})}+\mathrm{H}^{+}\right)$. A protonação deve ocorrer no átomo de oxigênio da ligação Si-O-Al. Como na estrutura zeolítica o $\mathrm{Al}$ forma apenas quatro ligações, ele irá apresentar deficiência de elétrons e agirá portanto, como sítio ácido de Lewis. Análises de difração de raios X da zeólita ZSM22 na forma amoniacal $\left(\mathrm{NH}_{4}{ }^{+} \mathrm{ZSM}-22\right)$ na direção (131) mostraram aumento de intensidade do pico correspondente, quando comparado com o padrão de difração da zeólita na forma protonada. Estes dados sugerem que os sítios ácidos de Brönsted encontram-se estatisticamente distribuídos da mesma forma que os sítios ácidos de Lewis, mas em quantidade mais pronunciada na direção (131) dos cristais.

O processo de ativação da zeólita ZSM-22 foi realizado por meio de termodecomposição do íon amônio presente na zeólita. Este processo foi estudado por meio do método de Ozawa, como previamente descrito. As curvas termogravimétricas (Fig. 3a) foram obtidas usando-se taxas de aquecimento de 2 a $10^{\circ} \mathrm{C} \cdot \mathrm{min}^{-1}$, apresentando regiões que puderam ser atribuídas à perda de água, em temperaturas inferiores a $250{ }^{\circ} \mathrm{C}$, assim como à decomposição do íon amônio, iniciando-se em $\sim 300{ }^{\circ} \mathrm{C}$. No processo de eliminação de água, verifica-se uma perda de cerca $4 \%$ de massa na análise realizada a $2{ }^{\circ} \mathrm{C} \cdot \mathrm{min}^{-1} \mathrm{o}$ que corresponde a 2,2 mmol de água por grama de zeólita. A perda de massa calculada a partir das demais análises, isto é, com maiores taxas de aquecimento não ultrapassou $1,7 \mathrm{mmol}$ de água por grama de zeólita. No gráfico do $\log \beta$ (taxa de aquecimento) em função de $1 /$ T (Fig. 3b), é observado um aumento gradual no coeficiente angular conforme há perda de massa, o que indica um decréscimo na energia de ativação necessária para que ocorra a eliminação de água presente na estrutura da zeólita. A termodecomposição dos íons amônio, que se dá a partir de $300{ }^{\circ} \mathrm{C}$, revela uma característica peculiar da estrutura. A energia de ativação calculada para este processo é $18,44 \mathrm{~kJ}^{\mathrm{mol}}{ }^{-1}$ e o fator pré-exponencial A é 2,2746. O baixo valor de A indica haver uma distribuição homogênea dos contra-íons na estrutura da zeólita ZSM-22 e, portanto, espera-se que os sítios ácidos também estejam distribuídos de forma ordenada. A estabilidade apresentada por estes íons pode ser explicada pelo fato de a energia de ativação permanecer constante mesmo com o aumento de temperatura (Fig. 3b), o que possivelmente está relacionado à estabilidade da estrutura zeolítica. Desta forma, esperase que os íons amônio estejam presentes em ambientes

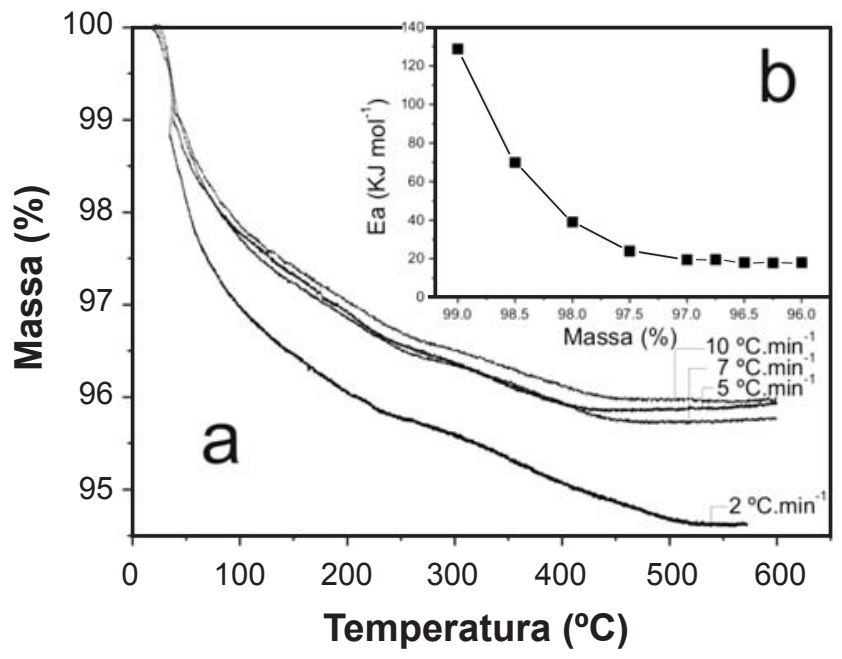

Figura 3: a) Curvas termogravimétricas da zeólita ZSM-22 na forma amoniacal a diferentes taxas de aquecimento ao ar; b) evolução da energia de ativação durante o processo de termodegradação da água e do íon amônio.

[Figure 3: a) Thermogravimetric curves of the ZSM-22 zeolite in the ammonium form at different heating rates in air and b) Activation energy as function of the residual weight.]

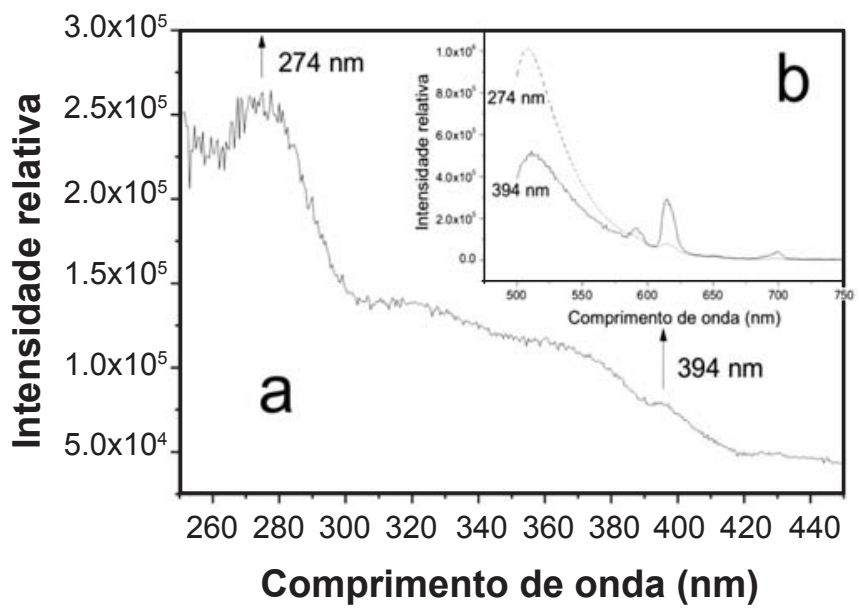

Figura 4: Espectros de a) excitação da amostra $\mathrm{Eu}^{3+} / \mathrm{ZSM}-22, \lambda_{\text {exc }}$ $274 \mathrm{~nm}$ e $394 \mathrm{~nm}$ e b) de emissão em $274 \mathrm{~nm}$ e $394 \mathrm{~nm}$.

[Figure 4: Excitation spectra for a) Eu ${ }^{3+} / Z S M-22$ sample, $\lambda_{\text {exI }} 274 \mathrm{~nm}$ and $394 \mathrm{~nm}$ and b) emission spectra for $\mathrm{Eu}^{3+} / \mathrm{ZSM}-22$ sample at 274 $\mathrm{nm}$ and $394 \mathrm{~nm}$.] 


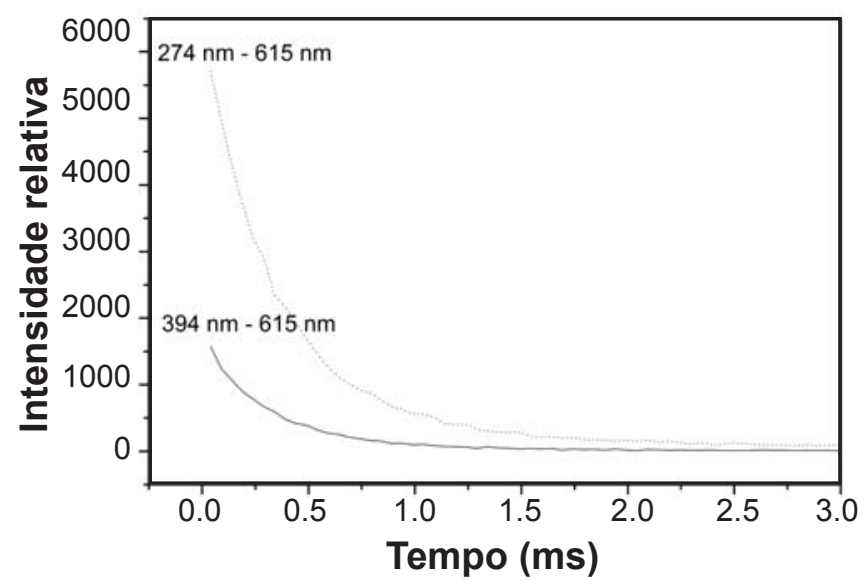

Figura 5: Curvas de decaimento da emissão $\mathrm{Eu}^{3+} \quad{ }^{5} \mathrm{D}_{0} \rightarrow{ }^{7} \mathrm{~F}_{2}$ da amostra $\mathrm{Eu}^{3+} / \mathrm{ZSM}-22$.

[Figure 5: Decay curves of the Eu ${ }^{3+}{ }^{5} \mathrm{D}_{0} \rightarrow{ }^{7} \mathrm{~F}_{2}$ emission in the sample $\left.E u_{3+} / Z S M-22.\right]$

químicos idênticos na estrutura zeolítica. Esta hipótese foi confirmada por meio de luminescência do európio, onde o íon $\mathrm{Eu}^{3+}$ foi usado como sonda, após substituir íons $\mathrm{NH}_{4}^{+}$. $\mathrm{O}$ espectro de excitação dos íons $\mathrm{Eu}^{3+}$ (Fig. 4a) mostra uma banda larga com centro em $274 \mathrm{~nm}$, a qual pode ser atribuída à transferência de carga do metal e pode estar relacionado à localização do íon. Acredita-se que os íons $\mathrm{Eu}^{3+}$ interajam majoritariamente com os oxigênios próximos aos grupos ácidos. A partir da emissão em 274 nm (Fig. 4b) é possível identificar a banda de transferência de carga no nível ${ }^{5} \mathrm{~L}_{6}$. Esta emissão quase desaparece completamente quando o íon é excitado a $394 \mathrm{~nm}$, quando a energia é provavelmente transferida para a matriz que emite luz em torno de $520 \mathrm{~nm}$.

Neste caso, a banda corresponde à transição ${ }^{5} \mathrm{D}_{0} \rightarrow{ }^{7} \mathrm{~F}_{2}$, a qual é mais forte que as transições ${ }^{7} \mathrm{~F}_{1}$ indicando que o íon ocupa sítios de baixa simetria. Novamente, bandas muito largas indicam uma ocupação não-homogênea dos sítios em que o íon $\mathrm{Eu}^{3+}$ se coordena. O decaimento da emissão ${ }^{7} \mathrm{~F}_{2} \rightarrow{ }^{5} \mathrm{D}_{0}$, em que os íons foram excitados em 394 e 274 nm (Fig. 5), indica a existência de mais de um sítio emissor. Os tempos de vida obtidos foram 0,24 e 0,69 ms para a excitação a $394 \mathrm{~nm}$ e 0,31 e 1,40 ms para excitação em $274 \mathrm{~nm}$. Estes tempos mostram que os íons podem estar perdendo energia para moléculas de água, para grupos $\mathrm{OH}$ ou para a matriz. Os baixos tempos de vida iniciais indicam um elevado número de moléculas de água ou grupos $\mathrm{OH}$ em torno do íon, o qual acarreta perda energética por meio de vibração da água.

Os espectros de infravermelho da zeólita ZSM-22 sem e com piridina, obtidos após desgaseificação em temperaturas crescentes são mostrados na Fig. 6. Os valores das áreas das bandas em 1450 e $1545 \mathrm{~cm}^{-1}$, características da interação entre a piridina e os sítios ácidos de Lewis e Brönsted respectivamente, foram calculadas e usadas para quantificar os sítios ácidos usando a Equação A.

As concentrações dos sítios ácidos de Brönsted e Lewis, determinadas após aquecimento a $100{ }^{\circ} \mathrm{C}$ sob vácuo foram: $\mathrm{q}_{\mathrm{B}}=173,12 \mu \mathrm{mol} \cdot \mathrm{g}^{-1}$ e $\mathrm{q}_{\mathrm{L}}=29,89 \mu \mathrm{mol} \cdot \mathrm{g}^{-1}, \mathrm{com} \mathrm{q}_{\mathrm{B}} / \mathrm{q}_{\mathrm{L}}=6$.

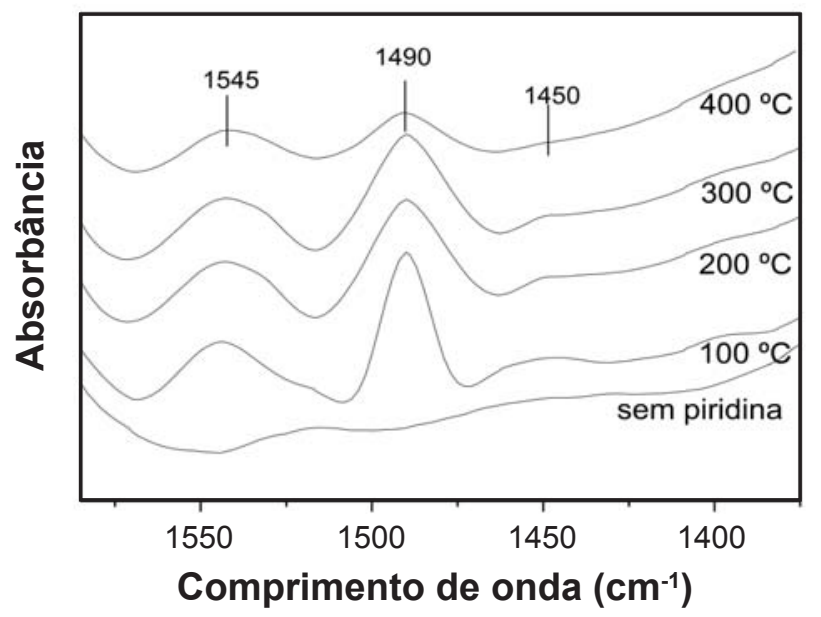

Figura 6: Espectros de infravermelho da zeólita ZSM-22 com piridina adsorvida obtidos após desgaseificação a diferentes temperaturas. As bandas observadas são decorrentes da interação da molécula de piridina com os sítios ácidos da zeólita.

[Figure 6: FTIR spectra of Pyridine (Py) adsorbed on ZSM-22 zeolite, recorded after outgassing at increasing temperatures. The observed bands are due to interactions between pyridine and the zeolite acid sites.]

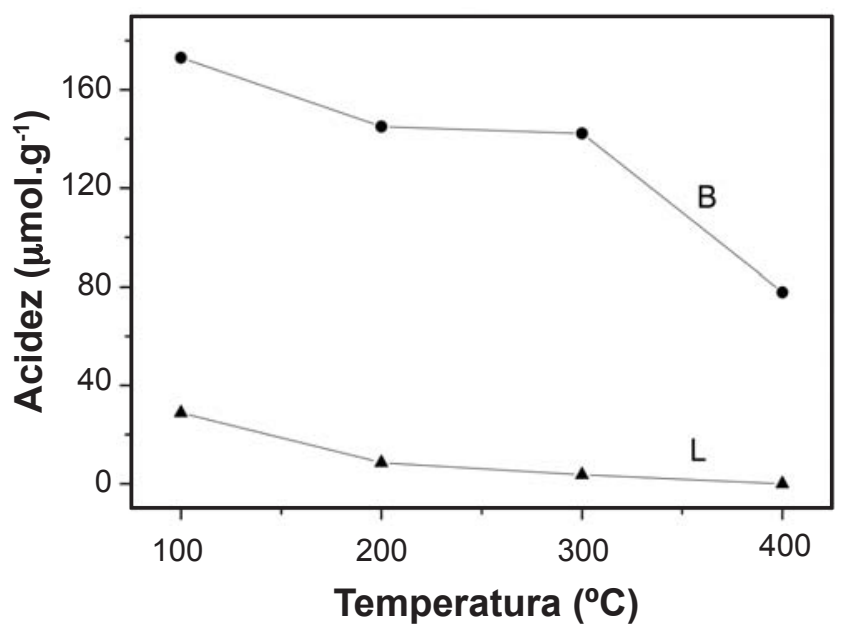

Figura 7: Acidez (B) de Brönsted e (L) de Lewis da zeólita ZSM22, determinada a partir das áreas das bandas observadas nos espectros de infravermelho em $1545 \mathrm{~cm}^{-1}$ e $1450 \mathrm{~cm}^{-1}$, em função da temperatura de desgaseificação da zeólita contendo piridina adsorvida.

[Figure 7: (B) Brönsted and (L) Lewis acidity of ZSM-22 zeolite, determined from the observed bands in the FTIR spectrum at 1545 $\mathrm{cm}^{-1} 1450 \mathrm{~cm}^{-1}$, as function of the outgassing temperature of the zeolite containing pyridine.]

A variação da intensidade relativa das bandas de Py-B e Py-L (piridina coordenada ao sítio ácido de Brönsted e de Lewis, respectivamente) em função das desgaseificações feitas com aumentos de temperatura é mostrada na Fig. 7. As informações extraídas deste gráfico são bons indicativos de que a acidez de Brönsted localiza-se no oxigênio ligado ao Si e $\mathrm{Al}$ ( $\mathrm{Si}-\mathrm{O}-\mathrm{Al}$ ), o que condiz com os resultados provenientes das análises de luminescência com $\mathrm{Eu}^{3+}$.

As medidas de adsorção-desorção de nitrogênio mostram uma isoterma do tipo I, característica de materiais 
microporosos. A zeólita apresenta área superficial $257 \mathrm{~m}^{2} \cdot \mathrm{g}^{-1}$, volume total de poro $0,053 \mathrm{~cm}^{3} \cdot \mathrm{g}^{-1}$, volume de microporo $0,041 \mathrm{~cm}^{3} \cdot \mathrm{g}^{-1}$ e diâmetro médio de poros $32,6 \AA$.

\section{CONCLUSÕES}

A zeólita ZSM-22 apresentou uma razão $\mathrm{Si} / \mathrm{Al}=46$, o que a faz um sólido poroso fortemente ácido mesmo em altas temperaturas $\left(\sim 400{ }^{\circ} \mathrm{C}\right)$. Esta zeólita apresenta acidez tanto de Lewis como de Brönsted, esta última em maior concentração. A energia de ativação, determinada pelo método de Ozawa, para os processos relacionados à ativação dos sítios ácidos de Brönsted foi $18,44 \mathrm{~kJ} \cdot \mathrm{mol}^{-1} \mathrm{e}$ o fator pré-exponencial 2,2746. A luminescência indicou uma distribuição uniforme de sítios ácidos. Sua estrutura é formada por microporos e apresenta área superficial de $257 \mathrm{~m}^{2} \cdot \mathrm{g}^{-1}$. Todas essas propriedades sugerem elevado potencial da ZSM-22 para uso em processos adsortivos e em catálise.

\section{AGRADECIMENTOS}

Ao CNPq e à CAPES pelo auxílio financeiro.

\section{REFERÊNCIAS}

[1] D. W. Breck, Zeolites: molecular sieves, $1^{\text {a }}$ Ed., John Wiley \& Sons, Inc, New York, EUA (1974).

[2] M. Guisnet, F. R. Ribeiro, Zeólitos: um nanomundo ao serviço da catálise, $1^{\mathrm{a}}$ Ed., Fundação Calouste Gulbenkian, Lisboa, Portugal (2004).

[3] K. Shams, S. J. Mirmohammadi, Micropor. Mesopor. Mater. 106 (2007) 268.

[4] C. S. Cundy, Chem. Rev. 103 (2003) 663.

[5] F. J. Luna, U. Schuchardt, Quim. Nova 24 (2001) 885.

[6] T. Demuth, X. Rozanska, L. Benco, J. Hafner, R. A. van Santen, H. Toulhoat, J. Catal. 214 (2003) 68.

[7] C. S. L. Narasimhan, J. W. Thybaut, G. B. Marin, Pierre A. Jacobs, J. A. Martens, J. F. Denayer, Gino V. Baron, J. Catal. 220 (2003) 399.

[8] R. Byggningsbacka, N. Kumar, L. E. Lindfors, J. Catal. 178 (1998) 611.

[9] F. J. del Rey-Perez-Caballero, G. Poncelet, Micropor. Mesopor. Mater. 37 (2000) 313.

[10] T. Ozawa, Bull. Chem. Soc. Jpn. 38 (1981) 1881.

[11] C. Busco, A. Barbaglia, M. Broyer, V. Bolis, G. M. Foddanu, P. Ugliengo, Thermochim. Acta 418 (2004)3.

(Rec. 27/11/2009, Ac. 20/02/2010) 